\title{
Short Stature in a Patient with Complex Rearrangement of Chromosome 1,4,11 and Growth Hormone Deficiency
}

\author{
Toru Kikuchi, Naoshi Hashimoto, Takuya Kawasaki,Hideo Takahashi and Makoto Uchiyama
}

Department of Pediatrics, Niigata University School of Medicine, Niigata, Japan

Key words: growth hormone deficiency, chromosomal abnormality, short stature

\section{Introduction}

Only a few chromosomal abnormalities with growth hormone (GH) deficiency such as abnormalities of chromosome 18,20,21, have been reported $(1,2,3)$. We report a case with a complex choromosomal rearrangement of 1 , 4, 11 with $\mathrm{GH}$ deficiency.

\section{Case Report}

The boy was first seen at 5.9 years of age because of proportional short stature. He was the product of a full-term uncomplicated pregnancy; birth weight was $2,680 \mathrm{~g}$. His parents were non-consanguineous. In the neonatal period, the patient received phototherapy. At the age of 3 months, he had Ramstedt's pyloromyotomy. His development was normal.

His height, weightand his bone age were $100.8 \mathrm{~cm}(-2.85 \mathrm{SD}), 17.8 \mathrm{~kg}(+22.8 \%), 3.7$ years old (BA/CA:55.5 \%) by TWII method (Japanese standard), respectively. His penis and pubic hair were prepubertal and his testis

Correspondence: Dr. Toru Kikuchi, Department of Pediatrics, Niigata University School of Medicine, 1-757, Asahicyou-dori, Niigata-shi, Niigata 951 Japan volumes were $1 \mathrm{ml}$. He had bilateral constricted ears, short neck and simian lines (Fig. 1).

Endocrinological investigations revealed that his GH was deficient(Table 1). Peak GH values after stimulation with insulin, clonidine and GRH were $6.8 \mathrm{ng} / \mathrm{mL}, 2.4 \mathrm{ng} / \mathrm{mL}, 13.4$ $\mathrm{ng} / \mathrm{mL}$, respectively. The mean value of spontaneous GH secretion during nocturnal sleep was $2.27 \mathrm{ng} / \mathrm{mL}$. Other pituitary functions and thyroid function were normal.

Chromosomal studies of his peripheral blood lymphocytes were performed (Fig. 2). He had reciprocal translocation of chromosomes 1 , 4,11 . The karyotype was $46, \mathrm{XY}, \mathrm{t}(1,4,11)$

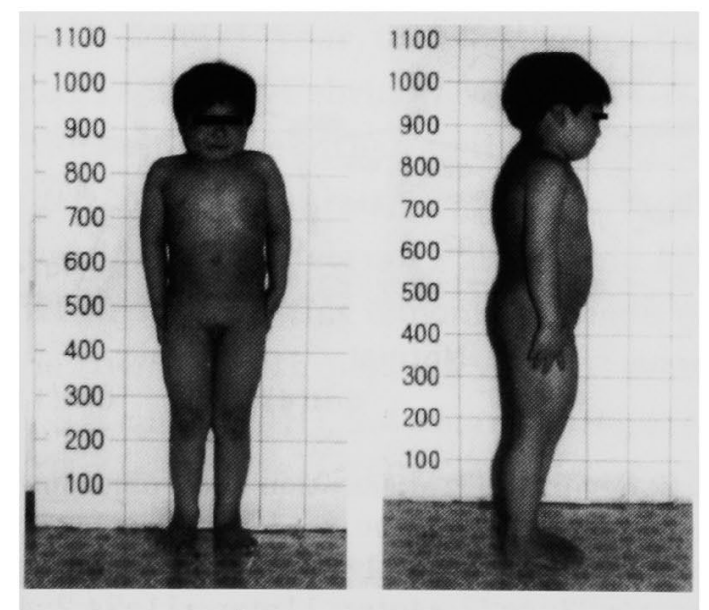

Fig. 1 The patient at the age of 6 


\section{Kikuchi et al.}

Table 1 Endocrinological findings

\begin{tabular}{|c|c|c|c|c|c|c|}
\hline \multicolumn{7}{|l|}{ minutes } \\
\hline $\mathrm{BS}(\mathrm{mg} / \mathrm{dl})$ & 87 & 26 & 49 & 67 & 72 & 79 \\
\hline $\mathrm{GH}(\mathrm{ng} / \mathrm{ml})$ & 0.2 & 0.2 & 4.4 & 6.2 & 6.8 & 1.5 \\
\hline ACTH $(\mathrm{pg} / \mathrm{ml})$ & 22.9 & 22.4 & 151.4 & 65.4 & 46.1 & 42.6 \\
\hline $\mathrm{TSH}(\mu \mathrm{U} / \mathrm{ml})$ & 1.8 & 13.0 & 10.1 & 7.8 & 6.1 & 4.0 \\
\hline $\mathrm{PRL}(\mathrm{ng} / \mathrm{ml})$ & 7.5 & 26.6 & 31.6 & 25.0 & 24.6 & 13.3 \\
\hline $\mathrm{LH}(\mathrm{mIU} / \mathrm{ml})$ & $<0.6$ & 0.6 & 1.1 & 0.9 & 0.8 & 0.6 \\
\hline $\mathrm{FSH}(\mathrm{mIU} / \mathrm{ml})$ & 0.8 & 2.9 & 4.6 & 4.8 & 5.4 & 4.8 \\
\hline \multicolumn{7}{|c|}{ Clonidine tolerance test } \\
\hline minutes & 0 & 30 & 60 & 90 & 120 & \\
\hline $\mathrm{GH}(\mathrm{ng} / \mathrm{ml})$ & 0.3 & 0.2 & 2.4 & 1.9 & 0.6 & \\
\hline \multicolumn{7}{|l|}{ GRF test } \\
\hline minutes & 0 & 15 & 30 & 60 & 90 & 120 \\
\hline GH (ng/ml) & 0.3 & 10.6 & 13.6 & 11.4 & 18.6 & 6.2 \\
\hline \multicolumn{7}{|c|}{ Nocturnal GH secration } \\
\hline minutes & 0 & 20 & 40 & 60 & 80 & 100 \\
\hline GH(ng/ml) & 0.35 & 4.60 & 5.21 & 3.97 & 1.89 & 0.78 \\
\hline minutes & 120 & 140 & 160 & 180 & mean & \\
\hline $\mathrm{GH}(\mathrm{ng} / \mathrm{ml})$ & 0.40 & 0.26 & 1.00 & 4.19 & 2.27 & \\
\hline IGF-I & $\mathrm{U} / \mathrm{ml}$ & & & & & \\
\hline 1.8 & $\mathbf{n g} / \mathbf{m l}$ & & & & & \\
\hline $\begin{array}{lr}\mathrm{T} 4 & 11.6 \\
\text { free T4 } & 1.6\end{array}$ & $\underset{\mathrm{ng} / \mathrm{dl}}{\mu \mathrm{g} / \mathrm{dl}}$ & & & & & \\
\hline
\end{tabular}

$(11 q$ ter $\rightarrow 11 q 24.2:: 1 p 36.11 \rightarrow 1 q 24.2:: 1 p 36.11 \rightarrow$ 1 pter $; 4$ pter $\rightarrow 4$ p23::4q27 $\rightarrow 4$ qter $; 11$ pter $\rightarrow$ $11 \mathrm{q} 24.2:: 4 \mathrm{q} 27 \rightarrow 4 \mathrm{p} 23:: 1 \mathrm{q} 24.3 \rightarrow 1 \mathrm{qter})$.

At the age of 6.6 years, GH therapy was started. The height velocity before $\mathrm{GH}$ therapy was $4.4 \mathrm{~cm} /$ year, and $9.7 \mathrm{~cm} /$ year after the first year. No adverse events have been observed.

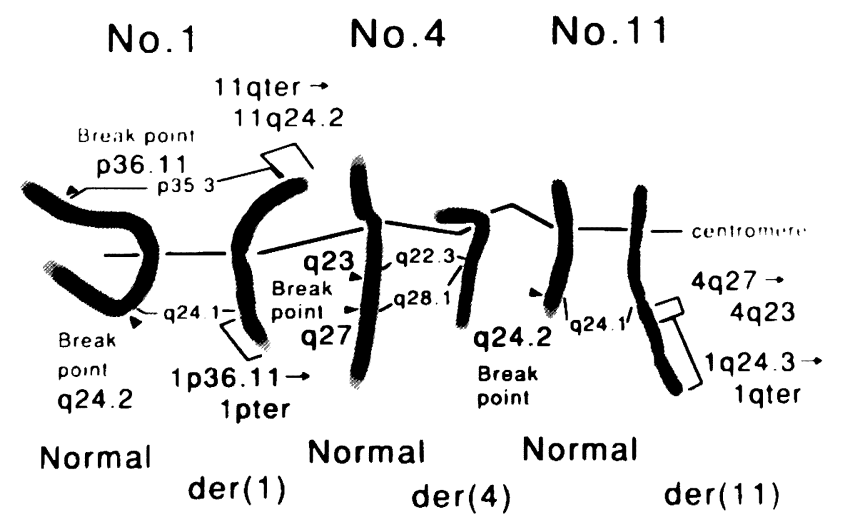

Fig. 2 Reciprocal translocation of chromosome 1,4 and 11. The karyotype is $46, \mathrm{XY}, \mathrm{t}(1,4,11)$ (llqter $\rightarrow 11 \mathrm{q} 24.2:: 1 \mathrm{p} 36.11 \rightarrow 1 \mathrm{q} 24.2:: 1 \mathrm{p} 36.11 \rightarrow 1$ pter; 4 pter $\rightarrow 4 \mathrm{p} 23:: 4 \mathrm{q} 27 \rightarrow 4 \mathrm{qter} ; 11 \mathrm{pter} \rightarrow 11 \mathrm{q} 24.2:: 4 \mathrm{q} 27$ $\rightarrow 4 \mathrm{p} 23:: 1 \mathrm{q} 24.3 \rightarrow 1 \mathrm{qter})$.

\section{Discussion}

Chromosome 18, 20, 21 abnormalities associated with $\mathrm{GH}$ deficiency $(1,2,3)$ have been reported. However, this is the first case with abnormalities of chromosome 1, 4, 11 associated GH deficiency. A causal association between GH deficiency and reciprocal translocation of chromosomes $1,4,11$ is difficult to justify in this case, because the gene for $\mathrm{GH}$ production lies on chromosome 17 and the degree of GH deficiency was partial. This case suggests that chromosomal abnormality may be associated with $\mathrm{GH}$ deficiency. Therefore GH deficiency should be sought for in cases of short stature with chromosomal abnormalities.

\section{References}

1. Abusrewil SS, Mcdermott A, Savage DCL. Growth hormone, suspected gonadotropin deficiency, and ring 18 chromosome. Arch Dis Child 1988; 63: 1090-1.

2. Schwart HP, Duck SC. Growth hormone deficiency in children with chromosome abnormalities. Arch Dis Child 1990; 65: 334.

3. Larizza D, Maraschio P, Maghnie M, Sampao1o P. Hypogonadism in a patient with balanced X/18 translocationand pituitary hormone deficiency. Eur J Pediatr 1993; 152: 424-7. 